\title{
LES FACTEURS SOCIAUX DES CARRIĖRES POLITIQUES DES FEMMES MINISTRES
}

\section{Une féminisation en trompe-l'œil}

\author{
Valentin Behr, Sébastien Michon
}

Belin | « Genèses »

2014/3 nº 96 | pages 132 à 156

ISSN 1155-3219

ISBN 9782701189918

DOI 10.3917/gen.096.0132

Article disponible en ligne à l'adresse :

https://www.cairn.info/revue-geneses-2014-3-page-132.htm

Distribution électronique Cairn.info pour Belin.

(C) Belin. Tous droits réservés pour tous pays.

La reproduction ou représentation de cet article, notamment par photocopie, n'est autorisée que dans les limites des conditions générales d'utilisation du site ou, le cas échéant, des conditions générales de la licence souscrite par votre établissement. Toute autre reproduction ou représentation, en tout ou partie, sous quelque forme et de quelque manière que ce soit, est interdite sauf accord préalable et écrit de l'éditeur, en dehors des cas prévus par la législation en vigueur en France. Il est précisé que son stockage dans une base de données est également interdit. 


\section{Les facteurs sociaux des carrières politiques des femmes ministres: \\ une féminisation en trompe-l'œil}

\section{Valentin Behr et Sébastien Michon}

PP. $132-156$

i, depuis la fin des années 1990 et la loi sur la parité (2001), le personnel politique s'est féminisé (Assemblée nationale, Sénat, exécutifs locaux), les femmes demeurent plus fréquemment cantonnées aux positions politiques les plus dominées (conseillers régionaux et municipaux) qu'elles n'accèdent aux positions les plus prestigieuses (députés et sénateurs) (cf. tableau 1). L'accès au gouvernement fait quelque peu exception, les derniers gouvernements étant plus féminisés que l'Assemblée et le Sénat. Certes, sous la Ve République, le métier de ministre demeure dans son ensemble un «métier d'hommes» (Achin 2005): au cours de la période 1986-2012 1 , 24\% des membres de gouvernement sont des femmes. Néanmoins, la présence des femmes au gouvernement a progressé de manière non négligeable, au point que le gouvernement apparait, depuis la fin des années 1990, comme plus féminisé que les deux chambres (cf. tableau 2). Quatre paliers peuvent être distingués. Entre 1958 et le début des années 1970, les femmes sont quasi exclues du gouvernement puisqu'on ne compte que deux femmes ministres. Entre 1981 et 1995, la part des femmes progresse - principalement du fait des gouvernements de gauche - et se situe entre $9 \%$ et $17 \%$ selon les gouvernements. Entre 1995 et 2012, à la suite du débat sur la parité, qui se fait entendre au moment de l'élection présidentielle de 1995 (Lagrave 2000), les femmes représentent un peu plus du quart des effectifs ministériels : 25,5\% dans le gouvernement Juppé 2 , 30\% dans le gouvernement Jospin, 28\% dans le gouvernement Fillon. Seul le gouvernement de Villepin, par ailleurs plus resserré sur le plan des effectifs, fait exception ${ }^{3}$. Si, habituellement, la féminisation du personnel politique est avant tout le fait des partis de gauche (Norris et Franklin 1997; 
Beauvallet et Michon 2008), la part des femmes ministres entre 1986 et 2012 est plutôt indifférenciée entre gauche et droite (26\% à gauche, $23 \%$ à droite). 되 Un nouveau palier est atteint par le gouvernement Ayrault, qui est le premier is gouvernement de la Ve République à assurer la parité entre hommes et femmes.

Tableau 1 : Participation des femmes à la Vie politique française

(Source: Observatoire de la parité, 2013)

\begin{tabular}{|l|c|c|}
\cline { 2 - 3 } \multicolumn{1}{c|}{} & Dernier scrutin & Part des femmes (EN \%) \\
\hline Conseillers municipaux & 2008 & 35,0 \\
\hline Maires (ensemble des communes) & 2008 & 13,8 \\
\hline Conseillers généraux & 2011 & 13,8 \\
\hline Présidents de conseils généraux & 2011 & 5,0 \\
\hline Conseillers régionaux & 2010 & 48,0 \\
\hline Présidents de conseils régionaux & 2010 & 7,7 \\
\hline Députés & 2012 & 26,9 \\
\hline Sénateurs & 2011 & 22,1 \\
\hline Parlementaires européens & 2009 & 44,4 \\
\hline
\end{tabular}

Tableau 2: Répartition homme-femme selon le gouvernement (1986-2012) (EN \%)

\begin{tabular}{|l|c|c|c|}
\hline \multicolumn{1}{|c|}{ Gouvernement } & Part des HOMmes & Part des Femmes & ENSEMBLE \\
\hline Chirac (1986-1988) $(\mathrm{N}=44)$ & 91 & 9 & 100 \\
\hline Rocard (1988-1991) $(\mathrm{N}=59)$ & 83 & 17 & 100 \\
\hline Cresson (1991-1992) $(\mathrm{N}=46)$ & 85 & 15 & 100 \\
\hline Bérégovoy (1992-1993) $(\mathrm{N}=47)$ & 85 & 15 & 100 \\
\hline Balladur (1993-1995) $(\mathrm{N}=32)$ & 91 & 9 & 100 \\
\hline Juppé (1995-1997) $(\mathrm{N}=47)$ & 74 & 26 & 100 \\
\hline Jospin $(1997-2002)(\mathrm{N}=48)$ & 71 & 29 & 100 \\
\hline Raffarin $(2002-2005)(\mathrm{N}=59)$ & 75 & 25 & 100 \\
\hline de Villepin (2005-2007) $(\mathrm{N}=33)$ & 82 & 18 & 100 \\
\hline Fillon $(2007-2012)(\mathrm{N}=68)$ & 72 & 28 & 100 \\
\hline Ayrault $(2012)(\mathrm{N}=40)^{4}$ & 52,5 & 47,5 & 100 \\
\hline Total (1986-2012) $(\mathrm{N}=349)$ & 76 & 24 & 100 \\
\hline
\end{tabular}


La promotion - relative - des femmes au gouvernement participe d'une mise en scène par les chefs de l'exécutif d'une bonne volonté à l'égard de la parité et de l'égalité homme-femme, et plus largement d'une forme de renouvellement du recrutement politique (Behr et Michon 2013). Les discours et commentaires sur la «crise» de la représentation politique n'ont donc pas été sans effet au centre du champ politique, le gouvernement étant devenu en quelque sorte une «vitrine de représentativité» (Sineau 2011 : 205-219). Si la parité semble devenue un remède à la crise de la représentation, un moyen de moderniser la vie politique à peu de frais (Achin 2001), sa mise en œuvre ne va pas sans poser problème aux dirigeants de l'exécutif lors de la formation des gouvernements. En mai 2007 par exemple, la presse évoque le dilemme de Nicolas Sarkozy, qui a promis lors de la campagne présidentielle de former un gouvernement strictement paritaire: «(..) il doit trouver, d'ici à la fin de la semaine, sept ou huit femmes. (...) Certes, il y a des candidates: outre Michèle Alliot-Marie, Christine Lagarde (...), Valérie Pécresse ou encore Rachida Dati. Mais beaucoup n'ont pas d'expérience gouvernementale, ce qui est un handicap pour tenir un gros ministère» (Le Figaro 2007). Au sein d'une institution comme le gouvernement, réputé accueillir des politiciens parmi les plus méritants et les plus aguerris, la moindre expérience politique des femmes est régulièrement pointée comme un handicap.

Un tel extrait de presse invite surtout à interroger les ressources qui favorisent les carrières des femmes au gouvernement sous la $V^{e}$ République, tant en termes d'accès au gouvernement que de carrières gouvernementales. Leurs profils sociaux et politiques sont-ils différents de ceux des hommes? Leurs carrières sont-elles plus rapides? Le critère partisan, qui ne distingue guère le recrutement social des élites politiques en général (Gaïti 1985), est-il plus heuristique dans le cas des femmes? Au sein des gouvernements de la $V^{e}$ République, les femmes sont-elles caractéristiques d'une ouverture du recrutement politique, ou au contraire des symboles d'une reproduction des élites? Une fois ministres, les femmes sont-elles cantonnées aux postes les plus subordonnés? Distingue-t-on des logiques genrées dans les accès au gouvernement et les carrières gouvernementales?

Dans la littérature spécialisée, les éléments de réponse à ces questions sont parcellaires. Si des travaux abordent le turnover en fonction de la popularité du gouvernement (Grossman 2009) ou de la dynamique des carrières (Kam et Indridason 2009), ils n'analysent pas les accès au gouvernement et les carrières gouvernementales au prisme du genre, et finalement très peu au regard des propriétés sociales. D'autres travaux plus anciens adoptent certes une perspective de sociologie politique essentiellement focalisée sur la problématique des filières d'accès au gouvernement (Dogan 1967; Gaxie 1986). En revanche, ils s'intéressent peu aux trajectoires gouvernementales, à la division du travail exécutif (Gaxie 1983) ou aux poursuites de carrières, et surtout ne tiennent pas compte du genre, tant la présence des femmes en politique était anecdotique à l'époque où ces recherches ont été menées. Enfin, la recherche de Catherine Achin et Sandrine Lévêque 
sur les femmes énarques et professionnelles de la politique (Achin et Lévêque 2007) apporte des éléments précieux sur la dynamique des carrières de plusieurs ministres et la mise en scène de leurs ressources politiques. Leurs résultats sont toutefois circonscrits à une minorité: sur la période 1986-2012, seules 14\% des femmes au gouvernement sont énarques. Aucune étude ne propose donc une analyse globale des trajectoires des ministres français au prisme du genre, qui lie les facteurs sociaux et politiques des accès au gouvernement et les trajectoires gouvernementales ${ }^{5}$. Pourtant, une entrée par le genre permet d'interroger les rapports entre ordre politique et ordre social (Lacroix 1985), entre légitimation du pouvoir politique - effet attendu de la féminisation - et préservation de l'ordre social - les femmes restant bien souvent cantonnées dans des rôles subalternes. La retraduction dans le champ politique de la problématique paritaire invite ainsi à «réfléchir sur l'autonomisation du politique, notamment dans ses rapports avec les principes de classement du monde social» (Dulong 2003 : 426).

Nous entendons combler ce manque grâce à l'analyse de deux bases de données prosopographiques des ministres français entre 1986 et 2012 (Premier ministre, ministre d'État, ministre, ministre délégué, secrétaire d'État). Outre la nécessité de ne pas essentialiser le critère du genre (Achin et al. 2007), l'analyse de ces données montre que la féminisation du personnel gouvernemental accompagne plus une reproduction qu'un renouvellement des élites politiques. La mise en perspective du genre au regard des capitaux sociaux et politiques des membres de gouvernement contribue à souligner le poids déterminant de ces capitaux non seulement sur l'accès au gouvernement, mais aussi sur les carrières gouvernementales des femmes.

Les bases de données ont été renseignées à partir d'informations extraites d'annuaires biographiques (Who's who in France). Étant donné les stratégies de présentation de soi pour ce type de données (Collovald 1988), les informations ont été croisées avec d'autres données disponibles en ligne (sites institutionnels, blogs, Wikipédia, articles de presse). La première base rassemble les 349 individus présents dans un ou plusieurs des onze gouvernements de la période étudiée: Chirac, Rocard, Cresson, Bérégovoy, Balladur, Juppé, Jospin, Raffarin, de Villepin, Fillon, Ayrault ${ }^{6}$. Les remaniements ministériels ont été pris en compte. Les profils des membres de gouvernement sont caractérisés par près de soixante-dix indicateurs de leurs propriétés sociodémographiques (année de naissance, genre, niveau et type d'études, institutions scolaires fréquentées, profession antérieure, etc.) et de leurs trajectoires politiques: mandats exercés et positions occupées (passage par une fonction de collaborateur politique, présence dans les gouvernements, nature du portefeuille gouvernemental, longévité aux différentes positions, etc.). La carrière de chaque individu a été retracée à partir de l'obtention du premier mandat (ou à défaut de l'entrée au gouvernement) jusqu'à la fin de l'année 2012 ou jusqu'à la fin de la carrière politique. La deuxième base liste l'ensemble des intitulés des postes gouvernementaux de ceux qui ont appartenu à au moins un gouvernement 
depuis 1986, ce qui représente 750 postes dont 655 postes sur la seule période 1986-2012. Ont été codés le type de poste, l'attribution et le rang protocolaire. Différents documents (autobiographies, blogs, articles de presse) ont également été consultés.

\section{Femmes au gouvernement: exceptionnelles, mais pas pour autant outsiders}

L'enquête permet d'éclairer des logiques du processus de féminisation du personnel politique au sommet et ses conséquences. La comparaison des propriétés sociales et politiques des ministres ${ }^{7}$ selon le critère du genre offre deux ensembles de résultats. D'un côté, les femmes ministres ne présentent pas des profils sociaux très différents de leurs homologues masculins: leur recrutement est lui aussi très élitiste socialement. La féminisation ne va donc pas de pair avec une ouverture sociale du recrutement politique. D'un autre côté, moins expérimentées en politique que les hommes, les femmes ministres se distinguent par un capital social, hérité ou acquis, non négligeable. Il s'agit d'une ressource qui entretient et perpétue le capital symbolique et peut ainsi favoriser leur carrière politique. En complément, l'étude des dynamiques de la féminisation au sein des gouvernements de gauche et de droite permet de rendre compte d'une relative hétérogénéité au sein du groupe des femmes.

\section{Sous le signe de la parité... la reproduction sociale}

Dire que le gouvernement recrute ses membres au sein d'une élite sociale (Gaxie 1983) et ce davantage que toute autre institution politique, est un constat qui reste d'actualité au cours des vingt-cinq dernières années : 80\% des ministres présents dans au moins un gouvernement depuis 1986 sont issus des classes supérieures $^{8}$. Ceci étant, les données rassemblées révèlent également que les parcours scolaires les plus prestigieux sont en recul depuis le début des années 2000: la part d'énarques au gouvernement se situe aujourd'hui à un niveau historiquement bas, à droite comme à gauche (Behr et Michon 2013). Dans ce contexte, qu'en est-il de l'origine sociale et des trajectoires scolaires des femmes ministres, dont la part s'est considérablement accrue depuis la fin des années 1990 ?

Sur le plan des trajectoires scolaires, les principaux écarts entre femmes et hommes ministres concernent le type d'études et leur prestige. Les femmes ministres ont plus souvent étudié en sciences humaines, en économie ou dans le domaine de la santé, mais nettement moins en sciences et techniques et surtout en sciences politiques. Ce dernier constat fait en partie écho à la moindre fréquentation par les femmes des cursus les plus légitimes: elles sont moins souvent passées par une grande école $(35 \%$ contre $47 \%)$, notamment par l'ENA (14\% contre $19 \%)$ et/ou Sciences Po Paris (25\% contre 30\%). 
Si les différences observées reflètent le plus souvent, bien qu'imparfaitement, des tendances qui traversent la société dans son ensemble (Achin 2005) - les femmes occupent des positions plus dominées dans la division du travail et suivent des cursus universitaires plus littéraires que scientifiques -, les trajectoires sociales et scolaires des femmes ministres sont tout de même très proches de celles de leurs homologues masculins. De facto, l'origine sociale des femmes ministres est comparable à celle des hommes: $76 \%$ d'entre elles sont issues des catégories supérieures de l'espace social contre $81 \%$ des hommes. Ceci va de pair avec des niveaux de diplôme assez similaires: $71 \%$ d'entre elles détiennent un diplôme au moins égal à bac +5 (contre $72 \%$ des hommes). Les femmes sont même un peu plus souvent titulaires d'un doctorat ( $24 \%$ contre $17 \%$ des hommes). Le croisement du critère du genre et du critère partisan interroge toutefois l'homogénéité du groupe des femmes. Leurs trajectoires scolaires sont bien plus différenciées entre gauche et droite que celles des hommes. Les femmes de droite sont davantage diplômées en droit (43,5\% d'entre elles) que les hommes de droite (35\%) et de gauche (30\%), et surtout que les femmes de gauche (18\%). À l'inverse, les femmes de gauche ont plus souvent étudié en sciences humaines (44\% d'entre elles) que les hommes de gauche (13\%) et de droite (19\%), et surtout que les femmes de droite (13\%).

Tableau 3: Origine sociale des membres de gouvernement selon le genre et l'orientation politique (en \%)

\begin{tabular}{|c|c|c|c|c|c|c|}
\hline & \multicolumn{3}{|c|}{ Femmes } & \multicolumn{3}{|c|}{ HoMmes } \\
\hline & $\begin{array}{l}\text { GAUCHE } \\
(\mathrm{N}=39)\end{array}$ & $\begin{array}{l}\text { DROITE } \\
(\mathrm{N}=46)\end{array}$ & $\begin{array}{l}\text { ENSEMBLE } \\
(\mathrm{N}=85)\end{array}$ & $\begin{array}{l}\text { Gauche } \\
(\mathrm{N}=111)\end{array}$ & $\begin{array}{c}\text { DroITE } \\
(\mathrm{N}=153)\end{array}$ & $\begin{array}{l}\text { ENSEMBLE } \\
(\mathrm{N}=\mathbf{2 6 4})\end{array}$ \\
\hline $\begin{array}{l}\text { Agriculteur, artisan } \\
\text { commerçant, chef d'entreprise }\end{array}$ & 3 & 0 & 1 & 6 & 11 & 9 \\
\hline Profession libérale & 11 & 22 & 16 & 9 & 14 & 12 \\
\hline Haut fonctionnaire & 26 & 15 & 20 & 18 & 24 & 22 \\
\hline Cadre supérieur du public & 18 & 27 & 23 & 37 & 18 & 26 \\
\hline Cadre supérieur du privé & 5 & 17 & 12 & 10 & 18 & 14 \\
\hline Professions intermédiaires & 8 & 9 & 8 & 8 & 3 & 5 \\
\hline Employé & 3 & 4 & 4 & 4 & 0 & 2 \\
\hline Collaborateur politique & 18 & 4 & 11 & 6 & 9 & 8 \\
\hline Autre profession & 3 & 2 & 2 & 2 & 3 & 2 \\
\hline Réponse manquante & 5 & 0 & 2 & 1 & 1 & 1 \\
\hline ENSEMBLE & 100 & 100 & 100 & 100 & 100 & 100 \\
\hline
\end{tabular}

Le constat d'une relative homogénéité sociale des élites politiques, qui transcenderait le clivage partisan (Birnbaum 1985), apparaît ainsi plus recevable dans le cas des hommes; les trajectoires sociales des femmes ministres reflètent plutôt le 
maintien de différences sociologiques assez classiques entre gauche et droite. Cette interprétation est renforcée par l'examen des niveaux de diplôme, qui sont moins élevés à gauche $(64 \%$ de diplômées à bac +5 et au-delà pour les femmes, $65 \%$ pour les hommes) qu'à droite ( $76 \%$ pour les femmes et $77 \%$ pour les hommes). De manière contre-intuitive, les femmes de gauche sont plus souvent diplômées de l'ENA (21\% d'entre elles) que les hommes de gauche (15\%) et surtout que les femmes de droite (9\%), mais moins que les hommes de droite (22\%). Ce dernier constat témoigne de la forte hétérogénéité sociale du groupe des femmes, notamment à gauche où se côtoient des femmes fortement dotées en capitaux culturels (à l'image de Martine Aubry, diplômée de l'IEP de Paris et de l'ENA) et d'autres parmi les moins dotées (Delphine Batho, par exemple, n'est pas diplômée du supérieur). L'analyse des trajectoires politiques apporte des éléments complémentaires sur les logiques de sélection des femmes ministres.

\section{Des femmes moins dotées en capitaux politiques légitimes}

Les différences entre les femmes et les hommes apparaissent particulièrement significatives du point de vue des indicateurs de la trajectoire politique. En ce sens, les femmes ministres sont «exceptionnelles» (Achin et Lévêque 2007). Leurs carrières politiques sont plus courtes, moins diversifiées et moins prestigieuses que celles des hommes.

Elles ont généralement moins d'expérience des mandats les plus légitimes: $51 \%$ d'entre elles ont été députées (contre $70 \%$ des hommes), $7 \%$ sénatrices (contre 13\%), 12\% maires (contre 51\%), 26\% conseillères générales (contre 49\%). Les seules exceptions concernent les mandats de conseiller régional et d'eurodéputé, soit des mandats obtenus dans le cadre de scrutins de liste qui renforcent le poids des états-majors des partis politiques dans le processus de désignation des candidats (Nay 1998; Beauvallet et Michon 2008; Lefebvre et Marrel 2012).

Lors de leur première nomination au gouvernement, les femmes ont un peu moins d'expérience politique que les hommes. Si elles entrent en politique ${ }^{9}$ au même âge (38 ans en moyenne contre 39 ans), elles sont en revanche plus jeunes au moment de l'entrée au gouvernement (47,7 ans contre 50,1 ans). Logiquement, au cours de leurs carrières électives, plus courtes, elles ont acquis un nombre moyen de mandats inférieur à celui des hommes, qu'il s'agisse du mandat de député, considéré comme le plus prestigieux ( 0,8 en moyenne contre 2 pour les hommes), ou de mandats de type différent (député, sénateur, maire, conseiller général, régional, municipal: 2 contre 3,1 pour les hommes). Ces écarts importants rendent compte de carrières électives de moindre intensité au cours desquelles le cumul de différents mandats (qu'il soit simultané ou étalé dans le temps) est moins fréquent pour les femmes que pour les hommes (Sineau 2011).

Outre les expériences électives, d'autres indicateurs confirment l'hypothèse d'un moindre volume des capitaux politiques des femmes ministres comparativement à ceux de leurs homologues masculins. En proportion, les femmes sont 
moins passées par un cabinet ministériel (26\% contre 38\%) et ont moins souvent exercé des responsabilités au sein des partis ( $46 \%$ contre $55 \%$ ). Moins expérimentées, moins dotées en capitaux politiques, tant électifs, politico-administratifs que partisans, les femmes ministres occupent des positions moins centrales au sein du champ politique que leurs homologues masculins, ce qui peut se traduire par un déficit de notoriété et un moindre accès à certaines ressources essentielles dans la compétition politique (accès aux médias, moyens associés à la fonction).

C'est en particulier le cas des femmes de droite qui sont plus éloignées du centre du champ politique que celles de gauche. Il s'agit d'une conséquence de la moindre féminisation des élus des partis de droite, les premières femmes députées étant principalement issues des partis de gauche (Sineau 2011). Compte tenu de ce vivier de femmes élues moins important, la contrainte de féminisation s'est traduite par la promotion de femmes assez peu expérimentées en politique dans les gouvernements de droite.

Au moment de l'accès au gouvernement, les expériences électives des femmes de gauche sont moins négligeables que celles des femmes de droite (cf. tableau 4). C'est parmi les femmes de droite que se trouve la plus forte part de ministres n'ayant jamais été élues avant leur première nomination au gouvernement (28\%). Les femmes de gauche, entrées en politique plus jeunes que leurs homologues de droite (38,2 ans contre 39,7 ans), présentent une plus grande longévité en politique (9,3 ans contre 8,3 ans) et des expériences électives plus proches de celles des hommes: elles ont plus souvent été élues députées (61,5\% contre $41 \%$ pour leurs homologues de droite), sénatrices (10\% contre $4 \%$ ) ou encore eurodéputées (15\% contre $9 \%)$, et ont détenu davantage de mandats de type différent avant l'entrée au gouvernement $(2,3$ contre 1,7$)$. Ceci n'est pas anodin puisque la détention et le cumul de mandats locaux et nationaux contribuent à l'implantation dans un fief électoral et à la construction de la notabilité, en même temps qu'ils procurent des ressources déterminantes pour la carrière politique (Politix 1994).

Plus expérimentées que les femmes ministres de droite en termes de mandats électifs, les femmes ministres de gauche présentent plus fréquemment des expériences de collaboratrice politique (assistante parlementaire, membre de cabinet, etc.). De même, elles sont plus souvent issues du militantisme, au sein d'organisations de jeunesse des partis, du syndicalisme étudiant ou d'associations à caractère politique (type SOS Racisme), et ont plus fréquemment occupé des responsabilités partisanes (en tant que secrétaires nationales, membres du bureau ou du comité national). Qui plus est, les femmes de gauche, bien que moins dotées en expériences électives que les hommes, présentent davantage d'expériences militantes, de collaboratrices politiques ou de responsables de partis. Elles apparaissent plus insérées dans les réseaux politiques et partisans que les femmes de droite et sont plus souvent représentatives d'une filière de recrutement militante ou partisane, filière relativement spécifique à la gauche au début des années 1980 (Gaït 1985). 
Tableau 4 : Expériences politiques des membres de gouvernement Selon le GenRe et l'orientation politique (en \%)

\begin{tabular}{|c|c|c|c|c|c|c|}
\hline & \multicolumn{3}{|c|}{ Femmes } & \multicolumn{3}{|c|}{ HOMMES } \\
\hline & $\begin{array}{l}\text { GAUCHE } \\
(\mathrm{N}=39)\end{array}$ & $\begin{array}{l}\text { DroITE } \\
(\mathrm{N}=46)\end{array}$ & $\begin{array}{c}\text { ENSEMBLE } \\
(\mathrm{N}=85)\end{array}$ & $\begin{array}{c}\text { GAuche } \\
(\mathrm{N}=111)\end{array}$ & $\begin{array}{c}\text { DroITE } \\
(\mathrm{N}=153)\end{array}$ & $\begin{array}{l}\text { ENSEMBLE } \\
(\mathrm{N}=\mathbf{2 6 4})\end{array}$ \\
\hline Assemblée nationale & 62 & 4 & 50 & 65 & 75 & 71 \\
\hline Sénat & 10 & 4 & 7 & 13 & 14 & 13 \\
\hline Parlement européen & 15 & 9 & 12 & 14 & 11 & 12 \\
\hline Conseil régional & 36 & 37 & 37 & 36 & 39 & 38 \\
\hline Conseil général & 36 & 17 & 26 & 52 & 46 & 49 \\
\hline Mairie & 15 & 9 & 12 & 50 & 52 & 51 \\
\hline Conseil municipal & 62 & 50 & 55 & 73 & 77 & 75 \\
\hline Aucun mandat & 15 & 28 & 22 & 17 & 14 & 16 \\
\hline Collaborateur politique ${ }^{10}$ & 54 & 41 & 47 & 41 & 53 & 48 \\
\hline Cabinet ministériel & 23 & 26 & 25 & 30 & 41 & 36 \\
\hline Militantisme jeune & 26 & 0 & 12 & 23 & 12 & 17 \\
\hline Responsabilité partisane & 51 & 41 & 46 & 46 & 61 & 55 \\
\hline
\end{tabular}

De la sorte, la conjoncture paritaire a favorisé la promotion au gouvernement de femmes moins dotées en ressources politiques (phénomène que l'on retrouve dans d'autres arènes politiques: Beauvallet et Michon 2008; Sineau 2011), plus encore à droite qu'à gauche. Elle a conduit à la promotion, à gauche, de femmes issues des réseaux militants et partisans, à droite, de femmes moins implantées dans le champ politique. Nonobstant, à gauche comme à droite, les femmes primo-accédantes au gouvernement ne sont pas tout à fait des outsiders du champ politique. Outre des expériences électives et professionnelles en tant que collaboratrices et militantes, elles possèdent souvent un capital social qui rend compte d'une certaine proximité avec le centre du champ politique.

«Filles de», «femmes de», "protégées de»

Les femmes au gouvernement se distinguent par un capital social non négligeable et pertinent dans le champ politique, susceptible de constituer une ressource dans la carrière politique. Trois dimensions du capital social peuvent être mentionnées: le capital social familial hérité, le capital social acquis par le mariage, enfin le capital social développé au cours d'activités professionnelles en lien avec le champ politique.

Une part non négligeable des femmes ministres sont des héritières, filles d'hommes ou de femmes politiques ( 12 femmes sur 85 , soit $14 \%$, ont un père ou une mère investis en politique, contre $11 \%$ des hommes), pour une part non négligeable 
d'anciens ministres ou parlementaires ( 7 femmes sur 85 , soit $8 \%$ contre $5 \%$ des hommes). Socialisées de manière précoce au métier politique, certaines ont hérité des mandats politiques de leurs pères.

Plusieurs exemples peuvent être cités. Michèle Alliot-Marie est la fille de Bernard Marie, ancien maire de Biarritz et député des Pyrénées-Atlantiques. Elle est élue députée de ce même département pour la première fois en 1986, après avoir été la suppléante de son père de 1978 à 1981, et avant d'être élue, en 1989, au conseil municipal de Biarritz, toujours auprès de son père. Roselyne Bachelot est la fille de Jean Narquin, ancien résistant, conseiller municipal d'Angers et député gaulliste de Maine-et-Loire. C'est dans ce département qu'elle entame sa carrière politique, en étant élue conseillère générale en 1982 avant de succéder à son père à la députation en 1988. Martine Aubry est la fille de Jacques Delors, qui fut, entre autres, ministre de l'Économie et des Finances (1981-1984) puis président de la Commission européenne en 1985. Le père de Nathalie Kosciusko-Morizet, François Kosciusko-Morizet, est maire de Sèvres depuis 1995 et conseiller général des Hauts-de-Seine depuis 2006; son grand-père, Jacques Kosciusko-Morizet, fut maire et ambassadeur de France de 1957 à 1978; son arrière-grand-père, André Morizet, fut sénateur et conseiller général de la Seine, ainsi que maire de Boulogne-Billancourt. Si ces exemples sont les plus significatifs, les biographies des femmes ministres donnent à voir un ancrage familial militant qui, bien qu'il ne soit pas toujours aussi prestigieux, suggère que la socialisation politique de ces futures ministres fut précoce. Par exemple, Aurélie Filippetti est la fille d'un ancien mineur devenu maire communiste d'une petite ville et conseiller général de Moselle; les parents de Cécile Duflot, professeure du secondaire pour l'une, cheminot pour l'autre, étaient tous deux syndicalistes; les parents de Sylvia Pinel furent tous deux élus municipaux de bastions radicaux dans le Midi-Pyrénées.

Il convient cependant de considérer ces informations au prisme des «identités stratégiques» (Collovald 1988) comme l'illustre la mise en avant, dans les biographies officielles des ministres, du «militantisme» ou du «syndicalisme» de leurs parents, qui permet de légitimer a posteriori la professionnalisation dans le métier politique et de revendiquer un ancrage populaire. De ce point de vue, la mise en scène par Aurélie Filippetti de ses origines immigrées et ouvrières (fille et petitefille de mineurs d'origine italienne) doit sans doute beaucoup au repositionnement politique stratégique quelle opère au moment où elle brigue un mandat de député en Lorraine. D'abord militante chez les Verts, élue conseillère du $5^{\mathrm{e}}$ arrondissement de Paris en 2001, elle ne parvient pas à être élue députée de Paris en 2002. Elle publie l'année suivante un roman intitulé Les derniers jours de la classe ouvrière, dans lequel elle conte son histoire familiale en Lorraine, avant de quitter les Verts en 2006 lorsque la direction du parti lui refuse l'investiture dans la $7^{\mathrm{e}}$ circonscription de Meurthe-et-Moselle, qu'elle brigue pour les législatives. En 2007, elle intègre l'équipe de campagne de Ségolène Royal en vue de l'élection présidentielle, puis est élue députée de la $8^{\mathrm{e}}$ circonscription de Moselle sous l'étiquette PS. Ainsi, la 
mobilisation de ses origines populaires peut se comprendre comme l'activation de dispositions sociales propices à l'obtention d'un mandat de député en Lorraine.

L'héritage politique, lorsqu'il prend la forme de la transmission des mandats électifs au sein de la famille, assure la perpétuation de la lignée politique (Patriat 1992). Toutefois, la surface sociale et politique des ministres n'est pas seulement héritée, mais aussi développée par la conjugalité (mariage ou concubinage) (Jérôme 2013). Or, avoir «fait un bon mariage» ou être en couple avec un insider en politique - forme d'union politisée - est plus fréquent pour les femmes ministres que pour leurs homologues masculins.

Les trajectoires et les ressources des femmes ministres sont relativement proches de celles de leurs conjoints. À l'instar des femmes membres de l'élite dirigeante économique, elles ont tendance à se marier avec des hommes de même rang social (Rabier 2013). Il s'agit plus souvent de chefs d'entreprises, de hauts-fonctionnaires ou de médecins. Au contraire, les hommes ministres occupent généralement des positions plus élevées dans l'espace social et le champ politique que leurs conjointes. Leurs conjointes sont plus souvent journalistes, professionnelles de la communication ou collaboratrices politiques. Cette hypergamie féminine - le statut social des conjoints des femmes ministres apparaît généralement plus élevé que celui des conjointes des hommes ministres - ne constitue cependant pas une spécificité de la population enquêtée (Bozon et Héran, 2006). Les conjoints des femmes ministres sont en outre plus souvent "politisés» au sens de professionnels de la politique: $25 \%$ des conjoints des femmes sont ou ont été élus ou collaborateurs politiques, contre seulement $10 \%$ des conjointes de ministres. De plus, les conjoints et conjointes politisés des ministres noccupent pas les mêmes positions au sein du champ politique.

D'un côté, les conjointes occupent généralement des positions relativement dominées, recrutées comme collaboratrices par leur conjoint ministre (Xavier Darcos, Marc-Philippe Daubresse, Yves Jégo, Brice Lalonde, Jack Lang, Jean Puech, Nicolas Sarkozy), parlementaire (François Sauvadet) ou maire (Patrick Devedjian). La presse est une excellente source d'information pour identifier ces recrutements conjugaux, souvent présentés par les protagonistes comme un moyen de maintenir la relation de couple malgré les contraintes de la vie politique. Cependant, il peut s'agir également - et stratégiquement - de pratiquer une forme d'ubiquité politique pour les ministres cumulards. Sophie Devedjian, par exemple, devient directrice de cabinet du maire d'Antony (Hauts-de-Seine) lorsque son mari Patrick, nommé ministre délégué du gouvernement Raffarin (2002-2005), doit quitter cette fonction pour celle de deuxième adjoint; un journaliste titre alors: «Le système Devedjian à Antony: Sophie, l'indispensable moitié» (L'Express 2005).

D'un autre côté, les conjoints des femmes ministres positionnés dans le champ politique occupent des positions plus centrales que celles des femmes de ministres. Il s'agit le plus souvent de hauts-fonctionnaires ou de conseillers politiques situés à l'intersection des champs politique et administratif, passés par les cabinets ministériels. 
C'est le cas de l'époux de Najat Vallaud-Belkacem, directeur de cabinet d'Arnaud Montebourg en 2013. C'est également le cas des conjoints de Rama Yade (historien et membre du cabinet ministériel de Jean-Marie Bockel en 2007) et d'Élisabeth Guigou (universitaire, membre d'un cabinet de Michel Rocard puis haut-fonctionnaire). Il peut s'agir enfin de conjoints diplômés de l'ENA (souvent mariés à des femmes ministres elles-mêmes énarques), tels que ceux de Simone Veil (hautfonctionnaire, dirigeant de grandes entreprises et élu centriste d'Île-de-France), d'Anne-Marie Idrac (préfet puis inspecteur général des finances) ou de Fleur Pellerin (conseiller d'État, puis directeur de cabinet adjoint de Marylise Lebranchu en 2013).

Ces résultats permettent de souligner l'homogamie sociale au sein des couples des ministres et surtout le différentiel de ressources sociales entre conjoints, plus équilibré au sein des couples des femmes ministres.

La troisième dimension de capital social est celle conférée par un «mentor» politique en capacité d'apporter son soutien à la carrière. Les postes de collaborateurs, situés dans les coulisses de l'action publique, représentent des voies de professionnalisation politique, voire un «accélérateur de carrière» (Mathiot et Sawicki 1999). Ils forment des expériences socialisatrices au métier politique et aux jeux politiques et permettent de développer une insertion dans des réseaux partisans, un capital social rentable dans le champ politique. Le soutien d'un élu jouant le rôle de «mentor» ou de "parrain» politique, prégnant pour de nombreux hommes ministres, l'est particulièrement pour plusieurs femmes, d'autant que ce sont souvent les élus les plus centraux au sein du champ politique qui jouent ce rôle. Il semble que l'une des voies privilégiées de la féminisation de l'exécutif ait été la promotion de femmes issues des entourages des hommes politiques (équipes, cabinets). Le soutien d'un mentor permet de compenser la relative faiblesse initiale des capitaux électifs, que l'on retrouve davantage chez les femmes que chez les hommes. À ce titre, les (rares) femmes présentes au sein des entourages des présidents de la République peuvent espérer bénéficier d'une promotion au gouvernement. Le secrétariat général (SG) de l'Élysée apparaît ainsi comme une pépinière de futures ministres pour les femmes énarques qui l'ont fréquenté. Ce sont les cas, à l'époque Mitterrand, de Frédérique Bredin, passée par le cabinet de Jack Lang au ministère de la Culture (1984-1986), conseillère de l'Élysée (1986-1988) avant d'être élue députée en 1988, puis d'être nommée ministre de la Jeunesse et des Sports (1991-1993); d'Élisabeth Guigou, qui intègre le SG de l'Élysée (1982-1990) après un court passage au sein du cabinet de Jacques Delors, ministre de l'Économie et des Finances (1982), puis intègre le gouvernement en tant que ministre déléguée chargée des Affaires européennes (1990-1993); enfin de Ségolène Royal, au SG de l'Elysée de 1982 à 1988, élue députée en 1988 puis nommée ministre de l'Environnement (1992-1993). Ces trois femmes ont en commun des trajectoires politiques très similaires, indéniablement favorisées par leur passage à l'Élysée - François Mitterrand aurait joué un rôle décisif dans les investitures de Frédérique Bredin et Ségolène Royal lors des élections législatives. 
Elles sont également parvenues à se maintenir au sein du champ politique en étant plusieurs fois réélues dans leurs fonctions parlementaires et en intégrant, pour deux d'entre elles (Guigou et Royal) le gouvernement Jospin. La droite n'est pas en reste, comme l'illustrent différents exemples. Catherine Colonna, énarque, réputée proche de Jacques Chirac et Dominique de Villepin, est nommée ministre déléguée aux Affaires européennes (2005-2007) après une carrière au Ministère des Affaires étrangères et une position de porte-parole de l'Élysée (1995-2004). Valérie Pécresse, énarque, au SG de l'Élysée de 1998 à 2002, députée de 2002 à 2007, est nommée ministre de l'Enseignement supérieur et de la Recherche (2007-2011). $\mathrm{Ou}$ encore Chantal Jouanno, elle aussi énarque, conseillère de Nicolas Sarkozy au ministère de l'Intérieur (2003-2004 puis 2006-2007), au conseil général des Hauts-de-Seine (2004-2006) et enfin à l'Élysée (2007-2008), qui intègre le gouvernement en tant que secrétaire d'État à l'Écologie (2009-2010).

L'héritage, le mariage, ou encore la proximité avec un mentor politique apparaissent comme des stratégies d'accumulation du capital social favorisant une ascension politique et sociale. Apparaître comme «femme de ", «fille de» ou "protégée de » traduit à la fois une socialisation relativement précoce au métier politique, mais aussi une volonté de la part des dirigeants de l'exécutif de satisfaire certaines personnalités influentes ou de récompenser des amitiés passées en promouvant des femmes qui leurs sont proches.

Si la féminisation du personnel gouvernemental ne s'est pas traduite par une diversification sociale du recrutement des ministres, les femmes ministres se distinguent néanmoins de leurs homologues masculins par des expériences politiques moindres et la détention de mandats plus secondaires. Les différences les plus marquantes concernent les femmes de droite, moins dotées en capitaux politiques que leurs homologues masculins. Pour autant, ce déficit en ressources politiques semble compensé, pour certaines, par une forme de capital social politique. $\mathrm{Ce}$ constat souligne l'inégal accès des femmes au jeu politique et plus précisément au centre du champ politique (Achin et Lévêque 2007), et pose la question des effets de ces différences sur les trajectoires gouvernementales.

\section{Les trajectoires gouvernementales des femmes, reflets des inégalités de capitaux}

L'analyse des trajectoires gouvernementales forme une deuxième dimension d'étude du processus de féminisation du personnel politique. Les carrières gouvernementales moins prestigieuses des femmes sont à lier à la détention différentielle des capitaux favorables à une carrière politique de premier plan, mise en évidence précédemment. Les femmes qui atteignent les sommets gouvernementaux s'avèrent les plus dotées en capitaux scolaires et électifs, mais aussi et surtout en capitaux politiques de diverses formes, accumulés au cours d'expériences militantes, 
professionnelles, ou encore par l'héritage et le mariage. Il s'agit non seulement de membres d'une élite sociale passée par l'ENA et la haute fonction publique, mais également d'une élite politique rapidement investie dans les réseaux partisans et la compétition électorale.

\section{Des carrières féminines moins prestigieuses}

Le type de poste occupé, le rang protocolaire, la longévité et les attributions forment un ensemble d'indicateurs des carrières gouvernementales. Leur croisement avec le genre atteste d'une division genrée du travail exécutif: les femmes occupent plus souvent les positions les plus dominées dans la hiérarchie des postes et des attributions et leurs carrières sont moins longues et moins ascendantes.

Les femmes ont tout d'abord plus de chances de se voir confier des positions gouvernementales secondaires, rattachées à un ministre de tutelle, le plus souvent un homme. Sur la période étudiée, elles sont davantage nommées secrétaires d'État que les hommes (37\% des postes occupés par les femmes sont des postes de secrétaires d'État contre $28 \%$ des postes occupés par les hommes). Plus on sélève dans la hiérarchie gouvernementale, moins la part de femmes est importante : $38 \%$ des postes occupés par les femmes sont des postes de Premier ministre, ministre d'État ou ministre contre $48 \%$ des postes occupés par les hommes. La distribution de l'ordre protocolaire des postes de ministres confirme le moindre prestige des positions des femmes ${ }^{11}$. Les postes qu'elles occupent sont plus éloignés du sommet que ceux des hommes: 11,1 de moyenne dans l'ordre protocolaire ${ }^{12}$ contre $10,1^{2}$ pour ceux des hommes ${ }^{13}$.

Des différences de trajectoires selon le genre se retrouvent dans la répartition des portefeuilles ministériels. Les femmes, qui représentent $20 \%$ environ de l'ensemble des ministres, ministres d'État et premiers ministres depuis 1986, sont très peu nombreuses parmi les titulaires des principaux portefeuilles régaliens (Défense, Affaires étrangères, Intérieur, Économie-Finances) - c'est-à-dire les plus centraux dans la hiérarchie gouvernementale -, ou encore de ceux de la fonction publique et de l'agriculture, domaine particulièrement masculin. Michèle Alliot-Marie dont la longévité politique tranche avec la moyenne des femmes - est ainsi la seule à avoir occupé les portefeuilles de l'Intérieur, de la Défense et des Affaires étrangères. Édith Cresson est la seule femme ayant dirigé un gouvernement. Fille de haut-fonctionnaire, épouse d'un cadre de Peugeot, diplômée d'HEC-Jeunes Filles et titulaire d'un doctorat, sa carrière est typique de la filière partisane: adhérente au PS dès 1971, elle en gravit les échelons jusqu'à la direction nationale, est élue eurodéputée en 1979 puis, après plusieurs échecs aux législatives, députée en 1981. Elle se voit confier plusieurs portefeuilles ministériels au cours des deux mandats de François Mitterrand, jusqu'à sa nomination à Matignon en 1991. Parmi les ministères régaliens, le ministère de la Justice fait exception avec $33 \%$ de femmes sur la période étudiée (Taubira, Alliot-Marie, Dati, Lebranchu, Guigou). À l'instar de la distribution des délégations au sein des conseils municipaux 
et régionaux (Achin et al. 2007), les femmes occupent principalement les portefeuilles les moins prestigieux et ceux jugés plus féminins tels que l'emploi et les affaires sociales ( $27 \%$ de femmes), la culture (29\%) et surtout la jeunesse et les sports (55\%) et l'environnement (57\%).

Le nombre de postes occupés et la position la plus prestigieuse permettent enfin de caractériser plus précisément les trajectoires gouvernementales. Si les femmes occupent des postes moins prestigieux, elles sont également moins souvent rappelées lors d'un remaniement ou de la formation d'un nouveau gouvernement: 1,9 poste ministériel en moyenne pour les femmes contre 2,3 postes pour les hommes. La distribution des positions les plus élevées atteintes témoigne également des probabilités moins élevées pour les femmes de faire carrière au gouvernement. Elles sont $27 \%$ parmi ceux qui ont été au mieux ministres délégués et secrétaires d'État, 24\% parmi ceux qui ont été au mieux ministres et 11\% seulement parmi les premiers ministres et ministres d'État. Il en va de même pour le rang protocolaire le plus élevé atteint par les femmes (11,1 en moyenne $\left.{ }^{14}\right)$, inférieur à celui des hommes $\left(9,5\right.$ en moyenne $\left.{ }^{15}\right)$. Les hommes ont donc plus de chances d'avoir une carrière gouvernementale ascendante que les femmes.

Structurantes au niveau des accès, les variations entre gauche et droite le sont également pour les trajectoires gouvernementales. Les femmes présentes dans les gouvernements de droite s'avèrent plus dominées que leurs homologues de gauche. Elles sont moins souvent ministres (Premier ministre et ministres d'État inclus) que leurs homologues de gauche: $34 \%$ des postes dans les gouvernements de droite ont été occupés par des femmes, contre $44 \%$ dans les gouvernements de gauche. Seules $39 \%$ des femmes de droite ont atteint un poste de ministre (ministres d'État inclus) contre $54 \%$ des femmes de gauche. Les carrières gouvernementales des femmes de droite s'avèrent également moins longues qu'à gauche: $19 \%$ d'entre elles ont occupé plus de deux postes contre $28 \%$ à gauche. Les femmes de droite obtiennent donc des positions ministérielles plus dominées, ce y compris pour celles qui atteignent un poste de ministre, leur rang protocolaire le plus élevé au cours de leur carrière étant en moyenne de 11,8 contre 10,6 pour les femmes des gouvernements de gauche. La position plus dominée des femmes à l'entrée au gouvernement perdure donc au cours de leur carrière gouvernementale. L'analyse centrée sur les femmes et les variations de carrières entre elles permet de mettre à l'épreuve les facteurs explicatifs mis en exergue précédemment. 
Tableau 5 : Type de poste au gouvernement selon le genRe et l'orientation polttique

$\mathscr{1}$

(Champ: L'ensemble des postes gouvernementaux entre 1986 et 2012) (en \%)

\begin{tabular}{|c|c|c|c|c|c|c|c|}
\hline & \multicolumn{3}{|c|}{ Femmes } & \multicolumn{3}{|c|}{ HоMмES } & \multirow[t]{2}{*}{ Ensemble } \\
\hline & Gauche & Droite & TотAL & Gauche & Drolte & TотAL & \\
\hline Poste occupé & $(\mathrm{N}=65)$ & $(\mathrm{N}=\mathbf{8 1})$ & $(\mathrm{N}=146)$ & $(\mathrm{N}=212)$ & $(\mathrm{N}=299)$ & $(\mathrm{N}=511)$ & $(\mathrm{N}=657)$ \\
\hline $\begin{array}{l}\text { Premier ministre ou } \\
\text { ministre d'État }\end{array}$ & 2 & 4 & 3 & 9 & 5 & 7 & 6 \\
\hline Ministre & 42 & 30 & 35 & 38 & 42 & 41 & 39 \\
\hline Ministre délégué & 31 & 21 & 25 & 21 & 26 & 24 & 24 \\
\hline Secrétaire d'État & 26 & 46 & 37 & 32 & 26 & 28 & 30 \\
\hline Total général & 100 & 100 & 100 & 100 & 100 & 100 & 100 \\
\hline
\end{tabular}

Tableau 6: Répartition des principaux portefeuilles ministériels selon le genre (Champ:

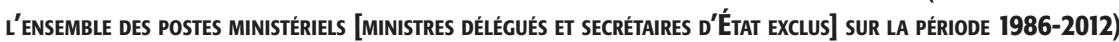

(Classement CROISSANT SUR LA COLONNE DE LA PART DE FEMmes)

\begin{tabular}{|c|c|c|c|c|c|c|}
\hline & & & & & & \\
\hline & EFfeCtifs & & Effectifs & & Effectifs & \\
\hline Économie Finances & 1 & 5 & 19 & 95 & 20 & 100 \\
\hline Intérieur & 1 & 5 & 19 & 95 & 20 & 100 \\
\hline Agriculture & 1 & 6 & 16 & 94 & 17 & 100 \\
\hline Fonction publique & 1 & 7 & 14 & 93 & 15 & 100 \\
\hline Affaires étrangères & 1 & 7 & 13 & 93 & 14 & 100 \\
\hline $\begin{array}{l}\text { Transports Logement } \\
\text { Équipement }\end{array}$ & 2 & 9 & 21 & 91 & 23 & 100 \\
\hline Éducation nationale & 2 & 9 & 20 & 91 & 22 & 100 \\
\hline Premier ministre & 1 & 9 & 10 & 91 & 11 & 100 \\
\hline Industrie Commerce & 2 & 12 & 15 & 88 & 17 & 100 \\
\hline Défense & 2 & 14 & 12 & 86 & 14 & 100 \\
\hline Budget & 1 & 17 & 5 & 83 & 6 & 100 \\
\hline Autre portefeuille & 8 & 24 & 26 & 76 & 34 & 100 \\
\hline Emploi Affaires sociales & 9 & 27 & 24 & 73 & 33 & 10 \\
\hline Culture & 4 & 29 & 10 & 71 & 14 & 100 \\
\hline Justice & 5 & 33 & 10 & 67 & 15 & 100 \\
\hline Jeunesse et Sports & 6 & 55 & 5 & 45 & 11 & 100 \\
\hline Environnement & 8 & 57 & 6 & 43 & 14 & 100 \\
\hline Ensemble & 55 & 18 & 245 & 82 & 300 & 100 \\
\hline
\end{tabular}


Tableau 7 : Nombre de postes ministériels et nOMbre d'anNÉEs PASSÉES Au GOUVERNement AU COURS DE LA CARRIĖRE SELON LE GENRE (EN \%)

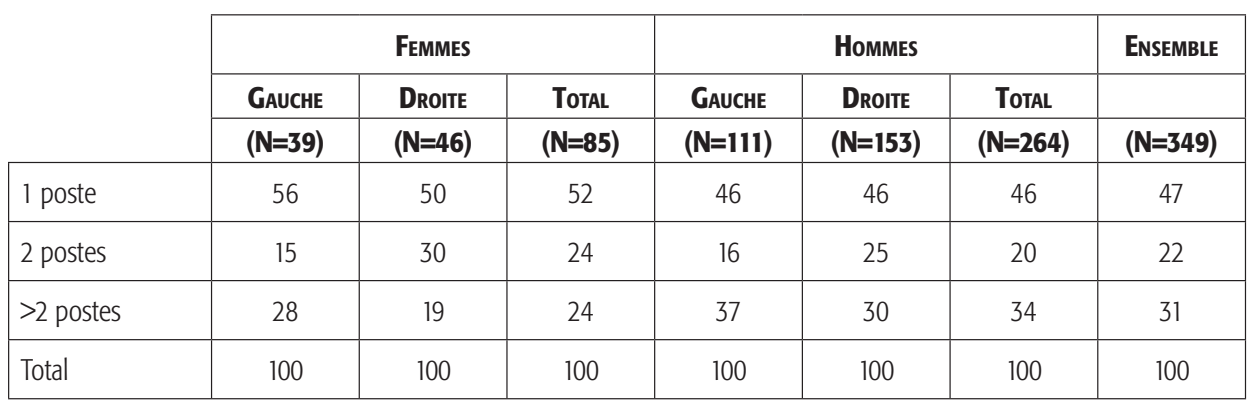

Tableau 8: Plus haut poste occupé et rang protocolaire LE PLUS Élevé SELON LE GeNRe et L'ORIeNTATION POLITIQUE (EN \%)

\begin{tabular}{|c|c|c|c|c|c|c|c|}
\hline & \multicolumn{3}{|c|}{ Femmes } & \multicolumn{3}{|c|}{ HOMMES } & \multirow[t]{2}{*}{ ENSEMBLE } \\
\hline & Gauche & Drolte & TOTAL & Gauche & DroIte & TOTAL & \\
\hline Plus haut poste occupé & $(\mathrm{N}=39)$ & $(\mathrm{N}=46)$ & $(\mathrm{N}=85)$ & $(\mathrm{N}=111)$ & $(\mathrm{N}=153)$ & $(\mathrm{N}=264)$ & $(\mathrm{N}=349)$ \\
\hline $\begin{array}{l}\text { Premier ministre ou ministre } \\
\text { d'État }\end{array}$ & 3 & 4 & 3,5 & 11 & 7 & 9 & 7,5 \\
\hline Ministre & 51 & 35 & 42,5 & 39 & 49 & 44,5 & 44 \\
\hline $\begin{array}{l}\text { Ministre délégué ou secrétaire } \\
\text { d'État }\end{array}$ & 46 & 61 & 54 & 50 & 44 & 46,5 & 48,5 \\
\hline Total général & 100 & 100 & 100 & 100 & 100 & 100 & 100 \\
\hline \multirow[t]{2}{*}{$\begin{array}{l}\text { Rang protocolaire le plus élevé } \\
\text { (moyenne) }\end{array}$} & $(\mathrm{N}=21)$ & $(\mathrm{N}=17)$ & $(\mathrm{N}=38)$ & $(\mathrm{N}=54)$ & $(\mathrm{N}=84)$ & $(\mathrm{N}=138)$ & $(\mathrm{N}=176)$ \\
\hline & 10,6 & 11,8 & 11,1 & 9,1 & 9,8 & 9,5 & 9,9 \\
\hline
\end{tabular}

Le poids des expériences sociales sur les trajectoires gouvernementales des femmes

À l'image des accès au gouvernement, les carrières gouvernementales ne sont pas équiprobables pour les femmes. L'étude des variations dans les carrières et de leurs facteurs explicatifs met en exergue des déterminants des carrières gouvernementales.

L'analyse des correspondances multiples (ACM), complétée par des tris croisés, constitue un outil heuristique pour mettre en évidence les liens entre les indicateurs de la carrière et l'espace des propriétés des ministres. La distinction entre variables actives et supplémentaires permet effectivement de projeter les caractéristiques de la carrière gouvernementale (placées en variables supplémentaires) sur l'espace des propriétés des femmes (constitué au moyen d'un ensemble de variables actives). 
L'ACM réalisée, menée sur les 85 femmes membres de gouvernement entre 1986 et 2012, représente l'espace des propriétés des femmes membres de gouvernement à partir d'indicateurs représentatifs de quatre dimensions des principales propriétés sociales et politiques évoquées précédemment: capitaux scolaires (passage par l'ENA et par Sciences Po), carrière élective (durée de la carrière, âge, passage par l'Assemblée nationale), expériences politiques non électives (collaborateur politique, militantisme de jeunesse, responsabilité partisane), capital social politique (fait d'avoir un conjoint investi en politique; un parent [mère ou - surtout - père] élu) ${ }^{16}$. Les caractéristiques de la carrière gouvernementale (plus haut poste et nombre de postes occupés, rang protocolaire maximal) et l'orientation politique ont été placées en variables supplémentaires: elles ne participent pas à la construction des axes de l'ACM. Les deux premiers axes forment un bon résumé de l'espace des propriétés (la somme des deux premiers taux modifiés de Benzécri ${ }^{17}$ atteint 95,2\%).

Tableau 9: Caractéristiques de l'ACM sur l'espace social et poltiloue des femmes ministres À LeUR ENTRÉE AU GOUVERNEMENT ( $\mathrm{N}=\mathbf{8 5}$, EFFECTIFS ENTRE PARENTHĖses)

\begin{tabular}{|c|c|}
\hline VARIABLES ACTIVES & $\begin{array}{l}\text { Capitaux scolaires légitimes (sur la figure 1, points représentés par un triangle) } \\
\text { - Diplômé de l'ENA: oui (12); non (73); } \\
\text { - Diplômé de Sciences Po Paris: oui (21); non (64); } \\
\text { Caractéristiques de la carrière élective (carré) } \\
\text { - Avoir été élu à l'Assemblée nationale (AN): oui (43); non (42); } \\
\text { - Durée de la carrière politique à l'entrée au gouvernement: } 0 \text { an (19); entre } 0 \\
\text { et } 10 \text { ans (36); supérieure à } 10 \text { ans (30); } \\
\text { - Âge au premier poste au gouvernement: avant } 40 \text { ans (18); entre } 40 \text { et } 50 \text { ans } \\
\text { (34); après } 50 \text { ans (33); } \\
\text { Expériences politiques non électives (croix) } \\
\text { - Avoir été collaborateur politique: oui (41), non (44); } \\
\text { - Avoir été militant dans des organisations de jeunesse: oui (11), non (74); } \\
\text { - Avoir eu des responsabilités partisanes: oui (39); non (46); } \\
\text { Capital social politique hérité ou acquis (losange) } \\
\text { - Conjoint en politique: oui (21); non (64); } \\
\text { - Père ou mère en politique: oui (12); non (73). }\end{array}$ \\
\hline VARIABLES SUPPLÉMENTAIRES & $\begin{array}{l}\text { Carrière gouvernementale (sur la figure } 2 \text { : carré vide) } \\
\text { - Plus haut poste occupé: ministre (y compris premier ministre et ministre d'État) } \\
\text { (40), ministre délégué ou secrétaire d'État (45); } \\
\text { - Nombre de postes au gouvernement: } 1 \text { poste (45); } 2 \text { postes (20); } \\
\text { plus de } 2 \text { postes (20); } \\
\text { - Rang protocolaire (variable continue) : } 38 \text { individus (moyenne de } 11,1, \\
\text { écart-type de } 6,0) \text {; } \\
\text { Orientation politique (triangle vide) } \\
\text { - Orientation politique: gauche (39), droite (46). }\end{array}$ \\
\hline
\end{tabular}




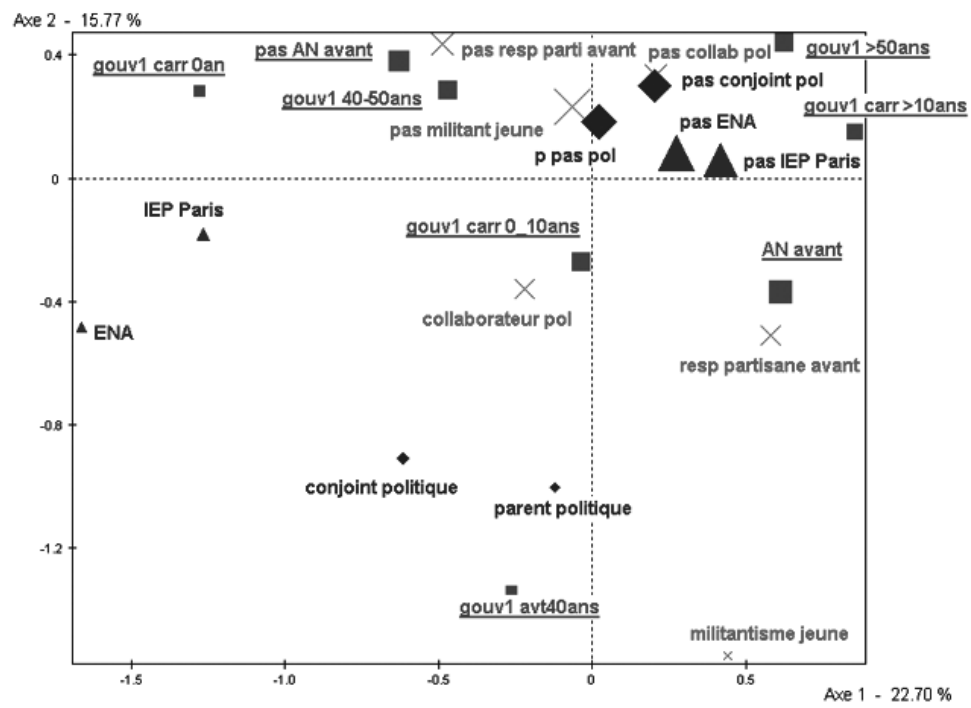

Figure 1: Analyse des correspondances multiples sur l'espace des femmes membres de gouvernement (1986-2012) ( $\mathrm{n}=85)$, projection des variables actives sur le plan formé par les axes 1 et 2 , taille des points proportionnelle au poids des modalités.

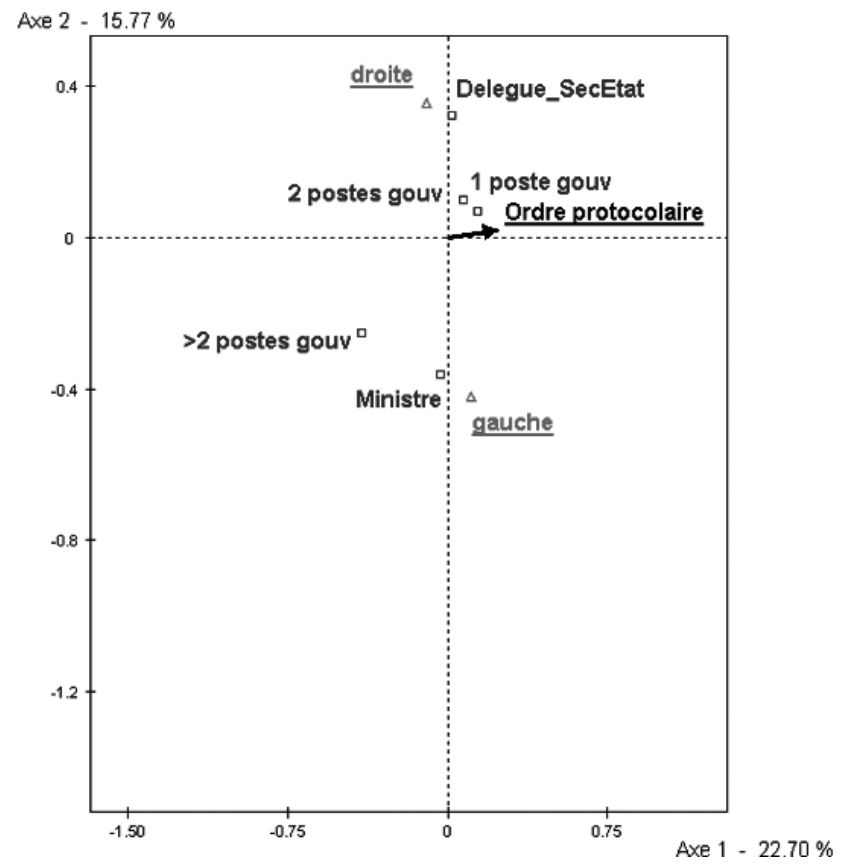

Figure 2: Analyse des correspondances multiples sur l'espace des femmes membres de gouvernement (1986-2012) ( $n=85)$, projection du positionnement politique, du nombre de postes, du poste le plus élevé et de l'ordre protocolaire maximal sur le plan formé par les axes 1 et $2^{18}$. 
Le premier axe $(22,70 \%$ de l'inertie totale, valeur propre 0,2724 , taux modifié $76,6 \%)$ distingue les entrées directes au centre de l'expérience politique, c'est-àdire celles qui entrent directement au gouvernement sans avoir été élues précédemment, de celles qui accèdent au gouvernement à la suite des carrières électives les plus longues (supérieures à 10 ans). Diplômées de l'ENA et/ou de Sciences Po, les premières ont plus souvent un conjoint investi en politique, assez souvent rencontré sur les bancs de Sciences Po ou de l'ENA. Moins dotées en capitaux scolaires, les secondes sont en revanche plus dotées en capitaux électifs et partisans: élues à l'Assemblée, elles ont eu des responsabilités partisanes et une expérience dans un mouvement politique de jeunesse. Le deuxième axe $(15,77 \%$ de l'inertie, valeur propre 0,1893 , taux modifié $18,6 \%$ ) est construit à partir des indicateurs de l'âge à l'entrée au gouvernement et des indicateurs d'expériences politiques non électives et de capital social. D'un côté (au sud du plan/cadran) se situent celles qui accèdent au gouvernement avant 40 ans, fortement dotées en capital partisan (responsabilité au sein du parti, militantisme au sein d'un mouvement de jeunesse) et en capital social hérité (parent en politique), acquis par le mariage ou le concubinage (conjoint en politique) ou développé dans le cadre d'activités politiques en tant que collaboratrice politique ou militante. De l'autre côté (au nord) se répartissent celles qui entrent au gouvernement après 50 ans et/ou qui sont les moins dotées en capital partisan et en capital social.

La projection des indicateurs de trajectoires gouvernementales sur le plan formé par les deux premiers axes de l'ACM confirme le positionnement plus dominé des femmes dans les gouvernements de droite et leurs plus faibles chances de connaître de longues et prestigieuses carrières ministérielles (cf. figure 2). Elle met également en exergue les variations de trajectoires des femmes ministres selon leurs capitaux scolaires et politiques. Ce ne sont pas tant les plus dotées en capitaux électifs que les plus dotées en capitaux scolaires et en capitaux politiques non électifs qui font les carrières gouvernementales les plus longues et les plus prestigieuses (sud-ouest).

D'une part, l'accès aux plus hautes fonctions ministérielles est plus probable pour celles qui détiennent les capitaux scolaires les plus légitimes ${ }^{19}$, ce qui rend compte d'une forme d'homologie entre l'espace des positions gouvernementales et l'espace social. Sur le plan constitué des deux premiers axes, le point «avoir occupé plus de deux postes au gouvernement» et le rang protocolaire le plus prestigieux (i.e. le plus bas, à lire en sens inverse de la flèche) se situent du côté des capitaux scolaires les plus légitimes. Plus précisément, les femmes qui atteignent le rang de ministre sont davantage formées au sein d'une grande école (50\% contre $22 \%$ pour les ministres délégués ou secrétaires d'État), particulièrement à l'ENA (20\% contre $9 \%)$ et/ou à Sciences Po (30\% contre 20\%). Les diplômées d'une grande école ont occupé en moyenne 2,2 postes de ministres (contre 1,6 pour les non diplômées) et émergent à un rang protocolaire maximal de 9,5 (contre 13,0) (respectivement 2,3 postes et un rang maximal de 9,4 pour les diplômées de l'ENA). 
L'accès plus fréquent aux positions les plus prestigieuses de celles qui sont issues de la «Noblesse d'État» (Bourdieu 1989) confirme leur recrutement élitaire via la filière politico-administrative, caractéristique de la Ve République (Dulong 2010).

D'autre part, plus que les capitaux électifs, ce sont les autres formes de capitaux politiques qui paraissent centrales dans les carrières politiques des femmes ministres: le point «ministre» (qui rassemble les ministres, les ministres d'État et les Premiers ministres) se situe sur le deuxième axe du côté des expériences politiques non électives et du capital social politique; quant au point relatif au cumul de plus de deux postes gouvernementaux, il est positionné dans le quart sud-ouest du graphique, du côté des femmes les plus dotées en capitaux scolaires légitimes (diplômées de l'ENA notamment) et en capital social politique (parent et conjoint en politique, expérience de collaboratrice, voire militantisme dans un mouvement de jeunesse).

Les activités politiques au sein du parti sont tout d'abord décisives: $50 \%$ des ministres ont eu des responsabilités partisanes avant leur premier accès au gouvernement (contre $42 \%$ des ministres déléguées et secrétaires d'État); 18\% d'entre elles ont milité dans des organisations de jeunesse (contre 9\%). Ces expériences permettent d'acquérir une connaissance fine des appareils et de leurs acteurs, de se positionner dans des courants ou autres tendances et d'acquérir du capital social susceptible de favoriser une cooptation (Bargel 2009).

Plus clivantes encore sont les expériences de collaboratrices politiques qui permettent une socialisation au métier politique et la construction d'un lien avec une personnalité politique, comme évoqué précédemment au sujet du rôle des mentors: $65 \%$ de celles qui ont occupé un poste de ministre ont travaillé pour des élus (contre $33 \%$ de celles qui ont occupé au mieux un poste de ministre déléguée ou de secrétaire d'État), spécialement au sein d'un cabinet ministériel (respectivement $40 \%$ contre $13 \%$ ); les anciennes collaboratrices politiques ont occupé en moyenne 2 postes ministériels (contre 1,6 sinon). Tel est particulièrement le cas des énarques, qui ont toutes été collaboratrices politiques avant d'entrer au gouvernement, notamment au sein d'un cabinet ministériel ou présidentiel (10 sur 12).

Enfin, un dernier ensemble de résultats a trait à la détention d'un capital social mobilisable dans le champ politique, obtenu par le mariage ou l'héritage. D'une part, $30 \%$ de celles qui ont été ministres ont un conjoint en politique (contre $20 \%$ des ministres déléguées ou secrétaires d'État); celles qui ont un conjoint en politique ont occupé 2 postes (contre 1,75) et surtout atteint un rang protocolaire maximal moyen de 7,8 (contre 12,7). D'autre part, $20 \%$ de celles qui ont été ministres ont un parent en politique (contre 11\% des ministres déléguées ou secrétaires d'État) et celles qui ont un parent en politique ont occupé 2,5 postes (contre 1,7). Ces ressources familiales s'avèrent une nouvelle fois plus fréquentes pour les énarques, mais également pour d'autres femmes un peu moins dotées en capitaux scolaires légitimes, mais en revanche davantage dotées en capitaux partisans (Alliot-Marie, Kosciusko-Morizet, Lienemann). 


\section{Conclusion}

Si la féminisation du recrutement politique, en particulier du recrutement gouvernemental, est indéniable, elle ne s'accompagne pas d'une transformation radicale ou même d'une «ouverture» de la composition sociale de l'exécutif. Les effets de la féminisation ne perturbent ni ne remettent en cause les modes de production et de reproduction des élites politiques. L'impression générale qui se dégage est plutôt celle d'une «reproduction dans la révolution» (Dulong 2003 : 431), comme l'illustrent la division du travail exécutif et l'occupation par les femmes des positions gouvernementales les plus secondaires ou dominées, de même que des portefeuilles se rattachant aux domaines considérés comme féminins (Affaires sociales, Jeunesse, Environnement, etc.).

Les résultats de l'enquête invitent à considérer les logiques différenciées d'accès aux positions de pouvoir entre hommes et femmes, et plus encore entre gauche et droite, au prisme des capitaux politiques détenus. Le genre peut être érigé en ressource politique par les professionnelles de la politique et se transformer en atout dans la compétition politique (Dulong et Lévêque 2002). Ceci étant, les femmes ministres ne sont pas des outsiders, symboles d'un renouvellement de la classe politique. Bien que moins expérimentées dans l'exercice des mandats et de la représentation, elles sont particulièrement dotées en capitaux politiques non électifs, notamment en capital social politique. La comparaison avec les hommes, puis une analyse centrée sur les femmes, montrent ainsi le rôle particulièrement décisif dans l'accès au gouvernement et dans le déroulement de la carrière gouvernementale de cette forme de capital social, hérité de père en fille, acquis par le concubinage ou développé au cours d'expériences de collaboratrices politiques auprès de personnalités parmi les plus centrales du champ politique, en mesure de les coopter pour accéder à des positions politiques (circonscription, poste ministériel). La féminisation du gouvernement se fait donc assez largement «en trompe-l'oeil», d'autant que les femmes, quoique progressivement mises en avant, demeurent exclues d'autres lieux d'exercice du pouvoir politique tels que les cabinets ministériels (Sineau 2011; Rouban 2013). 


\section{Ouvrages cités}

Achin, Catherine. 2001. «Représentation miroir" vs parité. Les débats parlementaires relatifs à la parité revus à la lumière des théories politiques de la représentation", Droit et société, $\mathrm{n}^{\circ}$ 47: 237-256.

— 2005. «Un "métier d'hommes”? Les représentations du métier de député à l'épreuve de sa féminisation ", Revue française de science politique, vol. 55, $\mathrm{n}^{\circ}$ 3: 477-499.

Achin, Catherine et Sandrine LÉvêQue. 2007. «Femmes, énarques et professionnelles de la politique. Des carrières exceptionnelles sous contraintes", Genèses, vol. 2, n 67 : 24-44.

Achin, Catherine et al. 2007. Sexes, genre et politique. Paris, Economica.

BARgel, Lucie. 2009. Jeunes socialistes/ jeunes UMP. Lieux et processus de socialisation politique. Paris, Dalloz.

Beauvallet, Willy et Sébastien Michon. 2008. «Les femmes au Parlement européen: effets du mode de scrutin, des stratégies et des ressources politiques. L'exemple de la délégation française ", Revue suisse de science politique, vol.14, n 4: 663-690.

Berr, Valentin et Sébastien Michon. 2013. "The Representativeness of French Cabinet Members, a Smokescreen?», French Politics, vol. $11, n^{\circ} 4: 332-355$.

Birnbaum, Pierre (éd.). 1985. Les élites socialistes au pouvoir, 1981-1985. Les dirigeants socialistes face à l'État. Paris, PUF.

Bourdieu, Pierre. 1989. La Noblesse d'État. Paris, Minuit.

Bozon, Michel et François Héran. 2006. La formation du couple. Textes essentiels pour la sociologie de la famille. Paris, La Découverte.

Collovald, Annie. 1988. «Identités stratégiques", Actes de la recherche en sciences sociales, $n^{\circ} 73: 29-40$.

Dogan, Mattéi. 1967. «Les filières de la carrière politique", Revue française de sociologie, vol. 8, $\mathrm{n}^{\circ} 5$ : 468-492.

Dulong, Delphine. 2003. «Des actes d'institution d'un genre particulier. Les conditions de légitimation des femmes sur la scène électorale (1945 et 2001)», in Jacques Lagroye (éd.), La politisation. Paris, Belin : 425-443.

Dulong, Delphine et Sandrine LÉvêQue. 2002. «Une ressource contingente. Les conditions de reconversion du genre en ressource politique », Politix, vol. 15, n 60 : 81-111.

Gaїтı, Brigitte. 1985. «Politique d'abord. Le chemin de la réussite ministérielle dans la France contemporaine », in Pierre Birnbaum (éd.) Les élites socialistes au pouvoir. Paris, PUF: 53-85.

Gaxie, Daniel. 1983. «Les facteurs sociaux de la carrière gouvernementale sous la Ve République 1958-1981 ", Revue française de sociologie, vol. 24, n 3: 441-460.

- 1986. «Immuables et changeants: les ministres de la $V^{e}$ République ", Pouvoirs, $n^{\circ} 36: 61-78$.

Grossman, Emiliano. 2009. «The President's Choice? Government and Cabinet Turnover under the Fifth Republic», West European Politics, vol. 32, n 2: 268-286.

Jérome, Vanessa. 2013. «"Nous nous sommes tant aimés”. (In)égalités des droits et questions sexuelles chez Europe écologie-Les Verts (EELV)», communication au Congrès de l'Association française de science politique, Paris.

KaM, Christopher et Indridi Indridason. 2009. «Cabinet Dynamics and Ministerial Careers in the French Fifth Republic», in Keith Dowding et Patrick Dumont (éd.), The Selection of Ministers in Europe. Londres, Routledge: 41-57.

Lacroix, Bernard. 1985. «Ordre politique et ordre social. Objectivisme, objectivation et analyse politique ", in Madeleine Grawitz et Jean Leca (éd.), Traité de science politique, vol. 1. Paris, PUF: 469-565.

LAgrave, Rose-Marie. 2000. «Une étrange défaite. La loi constitutionnelle sur la parité», Politix, vol. 13, n 51: 113-141. 
LE FIGARO, 2007. «Nicolas Sarkozy défend "l'ouverture" et prépare sa révolution gouvernementale», 15 mai.

Le Roux, Brigitte et Henry Rouanet. 2010. Multiple Correspondence Analysis. Quantitative Application in the Social Sciences. Londres, Sage.

Lefebvre, Rémi et Guillaume Marrel. 2012. «Logiques partisanes, territorialisation et capital politique européen. La constitution en France des listes socialistes aux élections européennes de 2009», Cultures et conflits, $\mathrm{n}^{\circ} 85-86: 139-162$.

L'EXPRESS, 2005. «Le système Devedjian à Antony: Sophie, l'indispensable moitié», 23 mai.

Mathiot, Pierre et Frédéric Sawicki. 1999. «Les membres des cabinets ministériels socialistes en France (19811993): recrutement et reconversion. 1) Caractéristiques sociales et filières de recrutement", Revue française de science politique, vol. 49, n 1:3-30.

Nay, Olivier. 1998. «Les règles du recrutement politique. Pour une approche institutionnaliste de la sélection politique», Politix, vol. 11, n 44: 161-190.
Norris, Pippa et Mark Franklin. 1997. $\mathscr{1}$ "Social Representation", European Journal of Political Research, vol. 32, $\mathrm{n}^{\circ}$ 2: 185-210.

Patriat, Claude. 1992. «Perspective cavalière. Où il est question de personnes éligibles naturellement et légitimement par voie d'héritage», in Claude Patriat et JeanLuc Parodi (éd.), L’hérédité en politique. Paris, Economica: 1-22.

PoLITIX. I 994. «Le métier d'élu. Jeux de rôles", vol. 7, n² 28.

Rabier, Marion. 2013. «Entrepreneuses de cause. Contribution à une sociologie des engagements des dirigeantes économiques en France ", thèse pour l'obtention du doctorat de science politique, EHESS.

Rouban, Luc. 2013. «L'accès des femmes aux postes de dirigeants de l'État», Revue française d'administration publique, $\mathrm{n}^{\circ} 145$ : 89-108.

Sineau, Mariette. 2011. Femmes et pouvoir sous la V République. De l'exclusion à l'entrée dans la course présidentielle. Paris, Presses de Sciences Po. 


\section{Notes}

1. Les bornes temporelles de notre étude (19862012) ont été principalement dictées par le constat - surprenant - de l'absence d'analyses exhaustives sur les membres de gouvernement depuis le milieu des années 1980 et les travaux de Daniel Gaxie entre autres. Pour autant, l'année 1986 ne correspond pas à une césure significative en termes de recrutement des ministres.

2.Même si la plupart d'entre elles ne sont restées au gouvernement que quelques mois.

3. Par commodité, on désigne par "gouvernement» l'ensemble des gouvernements formés par un même chef de gouvernement. Par exemple, l'expression "gouvernement Fillon» désigne l'ensemble des membres des gouvernements formés entre 2007 et 2012 .

4. Le gouvernement Ayrault est paritaire jusqu'à la démission de Jérôme Cahuzac si l'on effectue le comptage sans le poste de Premier ministre.

5. Il faut néanmoins rappeler que la faible présence des femmes au gouvernement jusqu'à une période récente rendait peu pertinentes des analyses statistiques en termes de genre. Le plus grand nombre de femmes ( 85 sur 349 membres de gouvernement) sur la période 1986-2012 permet d'intégrer cette variable dans l'analyse des voies d'accès au gouvernement et des carrières gouvernementales, et de mener des comparaisons plus systématiques avec la population masculine.

6. Au 31 décembre 2012.

7. Dans le corps du texte, le terme ministre est employé pour désigner l'ensemble des membres de gouvernement (secrétaire d'État, ministre délégué, ministre, ministre d'État, Premier ministre), sauf précision contraire.

8. Principalement cadres supérieurs du public (hauts fonctionnaires et enseignants) et du privé, mais aussi professions libérales (avocats et médecins) et, dans une moins mesure, grands patrons.
9. L'entrée en politique a été définie comme la date de l'obtention du premier mandat électif ou, à défaut, d'entrée au gouvernement.

10. Assistant parlementaire, membre cabinet ministériel, permanent d'un parti, etc.

11. Cet indicateur porte sur 290 postes de ministres sur les 300 recensés. Lordre protocolaire n'a pu être établi pour 10 ministres.

12. Moyenne calculée sur les 54 postes occupés par les femmes, médiane à 11 , écart-type de 6,1.

13. Moyenne calculée sur les 236 postes occupés par des hommes, médiane à 9 , écart-type de 5,9.

14. Moyenne calculée sur 38 personnes, médiane de 11, écart-type de 6,1. Lordre protocolaire maximal moyen est donc égal à l'ordre protocolaire moyen. Ce résultat surprenant s'explique entre autres par la proximité entre les deux distributions, et le nombre conséquent de postes centraux occupés par Michèle Alliot-Marie (quatre fois troisième et une fois sixième dans l'ordre protocolaire).

15. Moyenne calculée sur 176 personnes, médiane de 9,5, écart-type de 6,0.

\section{Soit 22 modalités.}

17. Sur la définition des taux modifiés de Benzécri: Le Roux et Rouanet 2010.

18. Lordre protocolaire maximal au cours de la carrière est une variable continue. Moins la valeur de l'ordre est élevée, plus le poste est prestigieux. De manière contre-intuitive, la flèche indique l'ordre protocolaire le plus éloigné du sommet du gouvernement.

19. Compte tenu des effectifs relativement modestes, l'analyse repose ici sur deux modalités synthétiques: d'un côté les ministres (premier ministre, ministres d'État et ministres, n=40), de l'autre les ministres déléguées et secrétaires d'État $(n=45)$. 